\title{
Validity and reliability of the session RPE method for monitoring exercise training intensity
}

\author{
L Herman (MS) ${ }^{1}$ \\ C Foster (PhD, FACSM) $)^{1}$ \\ M A Maher (PhD) $)^{2}$ \\ R P Mikat (PhD, FACSM) ${ }^{1}$ \\ J P Porcari (PhD, FACSM) ${ }^{1}$ \\ ${ }^{1}$ Department of Exercise and Sport Science, University of Wisconsin-La Crosse, USA \\ ${ }^{2}$ Department of Biology, University of Wisconsin-La Crosse, USA
}

\begin{abstract}
Objective. The Session Rating of Perceived Extertion (RPE) is a method of measuring exercise intensity that may be useful for the quantitative assessment of exercise training programmes. However, there are inadequate data regarding the validity and reliability of the Session RPE method. This study was designed to evaluate both the validity and reliability of the Session RPE method in comparison to objective measures $\left(\% \mathrm{HR}_{\text {peak }}, \% \mathrm{HR}_{\text {reserve }}\right.$ and $\left.\% \mathrm{VO}_{2 \text { peak }}\right)$ of exercise intensity.

Methods. Fourteen healthy volunteers ( 7 male, 7 female) performed 6 randomly ordered 30-minute constant-load exercise bouts at 3 different intensities, with each intensity being repeated. Oxygen consumption $\left(\mathrm{VO}_{2}\right)$ and heart rate $(\mathrm{HR})$ were measured throughout each exercise bout and normalised to maximal values obtained during a preliminary maximal exercise test. Thirty minutes following the conclusion of each exercise bout, the subject rated the global intensity of the bout using a modification of the Category Ratio (CR) (0 - 10) RPE scale. This rating was compared to the mean value of objectively measured exercise intensity across the duration of the bout.
\end{abstract}

Results. There were significant non-linear relationships between Session RPE and \%VO 2 peak $\left(\mathrm{R}^{2}=0.76\right), \% \mathrm{HR}_{\text {peak }}$ $\left(R^{2}=0.74\right)$ and $\% H R_{\text {reserve }}\left(R^{2}=0.71\right)$. There were no significant differences between test and retest values of $\% \mathrm{VO}_{2 \text { peak }}, \% \mathrm{HR}_{\text {peak }}, \% \mathrm{HR}_{\text {reserve }}$ and Session RPE during the easy ( 47 v. $47 \%, 65$ v. $66 \%, 47$ v. $48 \%$ and 2.0 v. 1.9), moderate (69 v. $70 \%, 83$ v. $84 \%, 74$ v. $75 \%$, and 4.2 v.

\section{CORRESPONDENCE:}

\section{Foster}

Department of Exercise and Sport Science

University of Wisconsin-La Crosse

La Crosse, WI 54601

Tel: 54601608-785 8687

Fax: 54601608-785 8172

E-mail: foster.carl@uwlax.edu
4.3 ) and hard (81 v. $81 \%, 94$ v. $94 \%, 91$ v. $91 \%$ and 7.3 v. 7.4) exercise bouts. Correlations between repeated bouts for $\% \mathrm{VO}_{2 \text { peak }}(r=0.98), \% \mathrm{HR}_{\text {peak }}(r=0.98), \% \mathrm{HR}_{\text {reserve }}(r$ $=0.98)$ and Session RPE $(r=0.88)$ were significant and strong.

Conclusions. The results support the validity and reliability of the Session RPE method of monitoring exercise intensity, although as might be predicted for a subjective method the Session RPE was less precise than the objective measures of exercise training intensity.

\section{Introduction}

The Session Rating of Perceived Exertion (RPE) method of monitoring exercise intensity was developed in $1995^{10}$ as a modification of the Category Ratio (CR) RPE method. 2,3,19,20,22 The modification involved asking the subject to give a global rating of the perceived exertion for the entire exercise session $\sim 30$ minutes after the conclusion of an exercise bout rather than rating the momentary level of exertion as is the usual practice with RPE. ${ }^{2,3,19,20,22}$ At that time, limited objective data were presented in support of the validity of the technique, on the basis of both heart rate $(\mathrm{HR})$ and blood lactate responses. In the subsequent decade, several papers have used the Session RPE method in a variety of settings and have demonstrated its value relative to quantifying exercise training intensities $s^{4,7,8,9,16,17,23}$ and as the intensity component of larger schemes of evaluating training programmes. ${ }^{6,7,10,11,13,21}$ Most notably, the Session RPE method has been used to demonstrate inconsistencies between the training programmes designed by coaches and executed by athletes, ${ }^{11}$ thus providing a plausible explanation for the incidence of overtraining syndrome in high-level athletes. ${ }^{6}$ However, the validity of the Session RPE method has not been systematically tested against accepted objective methods of measuring exercise training intensity, such as $\% \mathrm{VO}_{2 \text { peak }} \% \mathrm{HR}_{\text {peak }}$, and $\% \mathrm{HR}_{\text {reserve; }}$ nor has the reliability of the method been demonstrated. Accordingly, the purpose of this study was to evaluate the validity and reliability of the Session RPE method. 


\section{Methods}

The subjects were 14 physically active volunteers ( 7 males, 7 females). All subjects completed a health screening questionnaire ${ }^{1}$ which revealed no evidence that exercise testing or training should be limited. The subjects provided informed consent and the protocol for this study was approved by the Institutional Review Board for the Protection of Human Subjects at the University of Wisconsin-La Crosse. Characteristics of the subjects are presented in Table I. Since there was no expectation of a gender-related effect on the relationship between the Session RPE and objective markers of exercise intensity, the results of all subjects were pooled into a single group for analysis.

All subjects completed 7 different exercise sessions with a minimum of 2 days of rest between sessions. The first session was an incremental test to fatigue to determine maximal exercise responses for $\mathrm{HR}$ and peak oxygen uptake $\left(\mathrm{VO}_{2 \text { peak }}\right)$ (highest 30 -second $\mathrm{VO}_{2}$ observed during the test). Each subject completed all of their exercise sessions on either a cycle ergometer or treadmill, depending on their normal exercise habits. During the cycle ergometer protocol, the starting power output was $25 \mathrm{~W}$ and was increased by $25 \mathrm{~W}$ every 2 minutes. During the treadmill protocol, the belt velocity was set at either 5.6 or $8.1 \mathrm{kph}\left(1.56\right.$ or $\left.2.23 \mathrm{~m} . \mathrm{s}^{-1}\right)$, depending on whether the subject regularly walked or ran for exercise. The grade was $0 \%$ initially, and was increased by $2 \%$ every 2 minutes. The next 6 , randomly ordered, sessions, were performed using the same mode of exercise. Each was 30 minutes in duration, was of constant intensity within the trial, and varied in intensity between trials. There were 3 different intensity levels, designed to correspond to easy effort ( $40-50 \% \mathrm{VO}_{2 \text { peak }}$ ), moderate effort ( 60 - 70\% $\left.\mathrm{VO}_{2 \text { peak }}\right)$, and hard effort $\left(\sim 80-90 \% \mathrm{VO}_{2 \text { peak }}\right)$. Each subject completed each intensity session twice. During every exercise session, respiratory gas exchange was measured using open-circuit spirometry (Quinton Q-MC, Seattle, WA) and HR was measured using radiotelemetry (Polar ElectoOy, Finland). The RPE was assessed every 5 minutes throughout each exercise bout using the CR scale (Table II). Thirty minutes after completing the exercise session (after having time to shower and change clothes), each subject rated the perceived intensity of the entire exercise session according to the Session RPE scale (Table II), which is a modification of the conventional CR RPE scale, with verbal anchors changed to represent terms that are more relevant to exercisers fluent in American idiomatic English. In place of the usual specific verbal instructions normally used with

TABLE I. Mean ( \pm standard deviation) characteristics of the subjects

\begin{tabular}{|c|c|c|}
\hline & Men & Women \\
\hline Age (years) & $33 \pm 16$ & $23 \pm 1$ \\
\hline Height $(\mathrm{cm})$ & $180 \pm 9$ & $165 \pm 7$ \\
\hline Body mass (kg) & $93 \pm 17$ & $58 \pm 5$ \\
\hline VO2peak (ml.kg $\left.{ }^{-1}\right)$ & $41.5 \pm 9.9$ & $44.9 \pm 5.9$ \\
\hline HRpeak (beats.min ${ }^{-1}$ ) & $170 \pm 19$ & $186 \pm 22$ \\
\hline
\end{tabular}

\begin{tabular}{|c|c|}
\hline Rating & Verbal anchor \\
\hline 0 & Rest \\
\hline 1 & Very easy \\
\hline 2 & Easy \\
\hline 3 & Moderate \\
\hline 4 & Sort of hard \\
\hline 5 & Hard \\
\hline 6 & \\
\hline 7 & Very hard \\
\hline 8 & Very, very hard \\
\hline 9 & Near maximal \\
\hline 10 & Maximal \\
\hline
\end{tabular}

the RPE scale, the only verbal prompting was 'How was your workout?'

Statistical comparisons were made using repeated measures analysis of variance (ANOVA) for an intensity $x$ trials design. Statistical significance was accepted when $p$ $<0.05$. Post hoc comparisons, when justified by ANOVA, were performed using the Tukey test. Curve fitting and computation of correlation coefficients and/or coefficients of determination were made using a least squares technique.

\section{Results}

The tests were completed by all subjects without complications, and all subjects completed all tests. The mean serial responses of $\mathrm{VO}_{2}, \mathrm{HR}$ and $\mathrm{RPE}$ are presented in Fig. 1. The low-intensity exercise bout satisfied criteria for a steady state of $\mathrm{VO}_{2}$, whereas the moderate and hard exercise bouts displayed evidence of a slow component of $\mathrm{VO}_{2}$. All outcome measures differed $(p<0.05)$ between the 3 intensity levels. There was no significant difference between exercise intensity, measured by $\% \mathrm{VO}_{2 \text { peak }} \% \mathrm{HR}_{\text {peak }}, \% \mathrm{HR}_{\text {reserve }}$ and Session RPE during repeat trials at the same exercise intensity bout (Fig. 2).

The Session RPE estimate of exercise intensity was also shown to be reliable using regression analysis. The relationships between day 1 and day 2 for Session RPE $\left(R^{2}\right.$ $=0.78$, standard error of estimate $(\mathrm{SEE})=1.2), \% \mathrm{VO}_{2 \text { peak }}$ $\left(R^{2}=0.96\right.$, SEE $\left.=3.2\right), \% H R_{\text {peak }}\left(R^{2}=0.93\right.$, SEE = 3.7), and $\% H R_{\text {reserve }}\left(R^{2}=0.93\right.$, SEE $\left.=5.7\right)$ were statistically significant, and had a small SEE (Fig. 3).

The Session RPE method was compared with $\% \mathrm{VO}_{2 p e a k}$, $\% \mathrm{HR}_{\text {peak }}$ and $\% \mathrm{HR}_{\text {reserve }}$ to gain an appreciation of the degree to which the various methods of exercise intensity were measuring the same thing. The $\mathrm{R}^{2}$ between the Session RPE and $\% \mathrm{VO}_{\text {2peak }}\left(\mathrm{R}^{2}=0.76\right), \% H R_{\text {peak }}\left(\mathrm{R}^{2}=0.74\right)$, and $\% \mathrm{HR}_{\text {reserve }}$ $\left(R^{2}=0.71\right)$ was strong, but not comparable to the $R^{2}$ amongst the various objective measures of exercise intensity (Fig. 4). There was apparently a much weaker relationship between the Session RPE and the objective measures of exercise intensity at the higher exercise intensities (Fig. 4). 

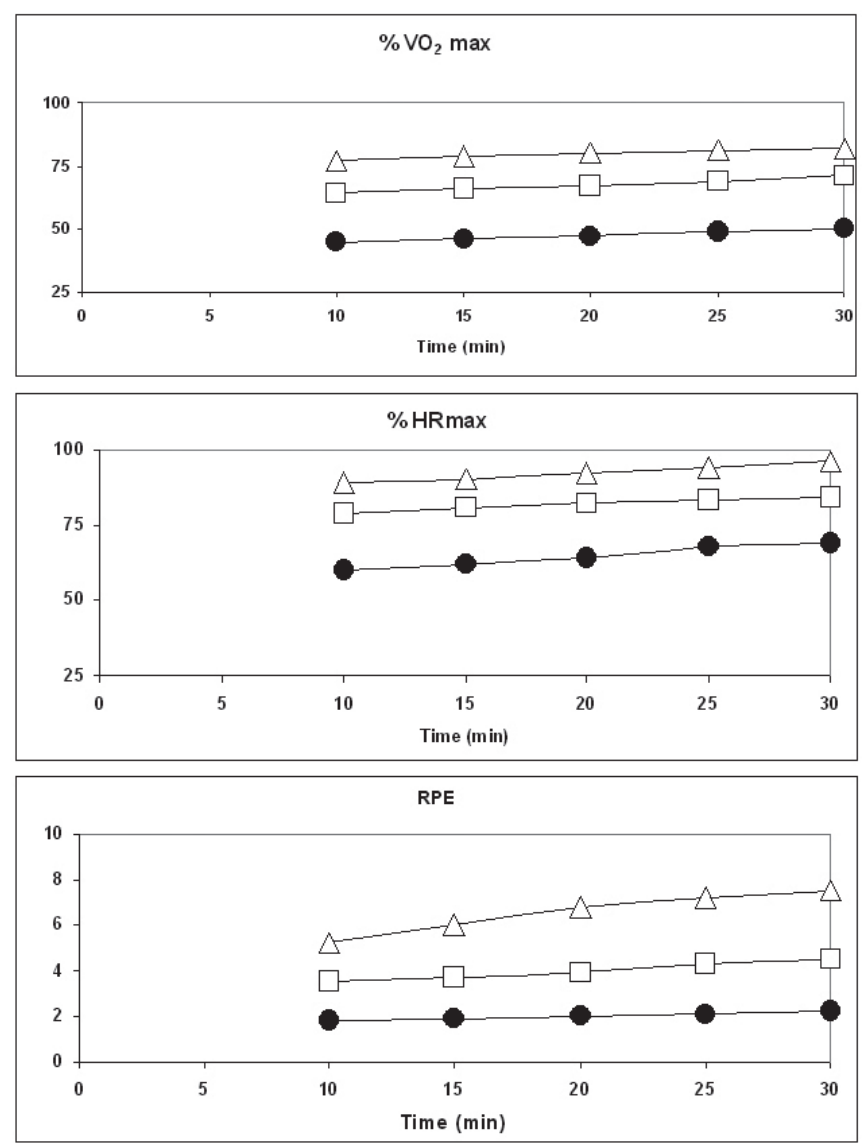

Fig. 1. Serial responses of $\% V_{2 \max }, \% H R_{\max }$ and $R P E$ during the course of the easy, medium and hard exercise bouts. The two trials at each intensity were combined to produce the figures.
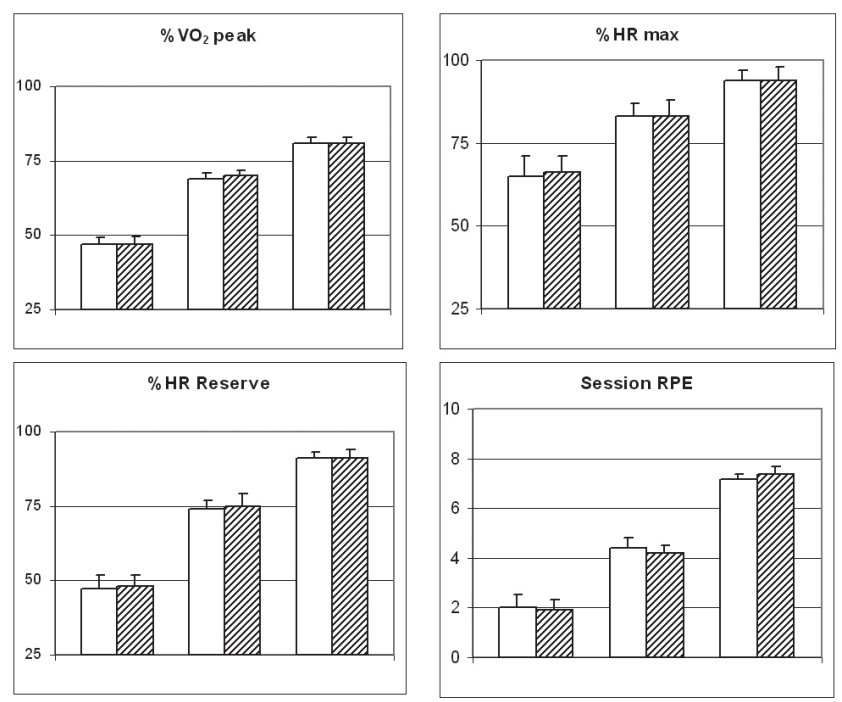

Fig. 2. Mean responses of $\% V \mathrm{O}_{2 \text { peak }} \% H R_{\max }, \% H R_{\text {reserve }}$ and Session RPE during Trial 1 and Trial 2 of the three exercise intensity bouts.
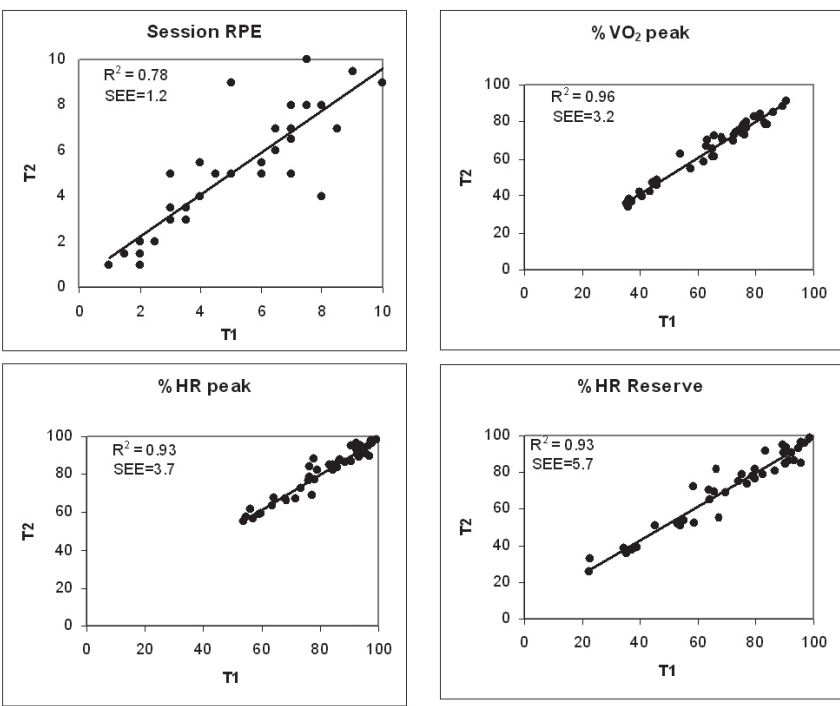

Fig. 3. Scatter plots of individual responses of Session

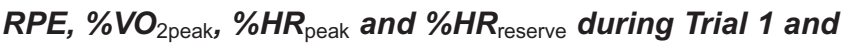
Trial 2, with all three intensities combined.

\section{Discussion}

The Session RPE has already been shown to be of value in terms of evaluating exercise intensity and monitoring training in a number of situations. ${ }^{6,7,10,11,13,21}$ The primary outcome of this study is the demonstration that the Session RPE method is not only reliable during repeat challenge by the same exercise stimulus, but is well related to widely accepted objective measures of exercise training intensity. As such, we interpret the present data as supporting the validity of the Session RPE method as an alternative method of monitoring exercise training. The present data are comparable with previous studies ${ }^{2,3,19,20,22}$ which have shown RPE to be a valid and reliable measure of momentary exercise intensity. We chose not to measure blood lactate responses during either incremental or constant intensity exercise, which is the other widely accepted objective method of measuring exercise intensity. This decision was based on practical concerns rather than any particular limitation of using lactate as a monitoring tool. Other studies demonstrating session RPE and lactate responses during similar exercise bouts would be of interest, and would provide a test of our early results with the Session RPE method compared with blood lactate responses. ${ }^{10}$

In this study, we used constant-load exercise bouts as the method for providing the exercise challenge. This was done because it was much more convenient to represent the exercise intensity using objective methods during steady-state exercise. Previous studies from our laboratory have compared the Session RPE method with HR-based measures of exercise intensity during non-steady state exercise, ${ }^{8,9}$ with substantially similar findings to the present data. Thus, we believe that the collective experience suggests that the Session RPE method is a valid marker of exercise intensity under a variety of situations. Other studies from our laboratory have demonstrated that the 'drift' in Session RPE 

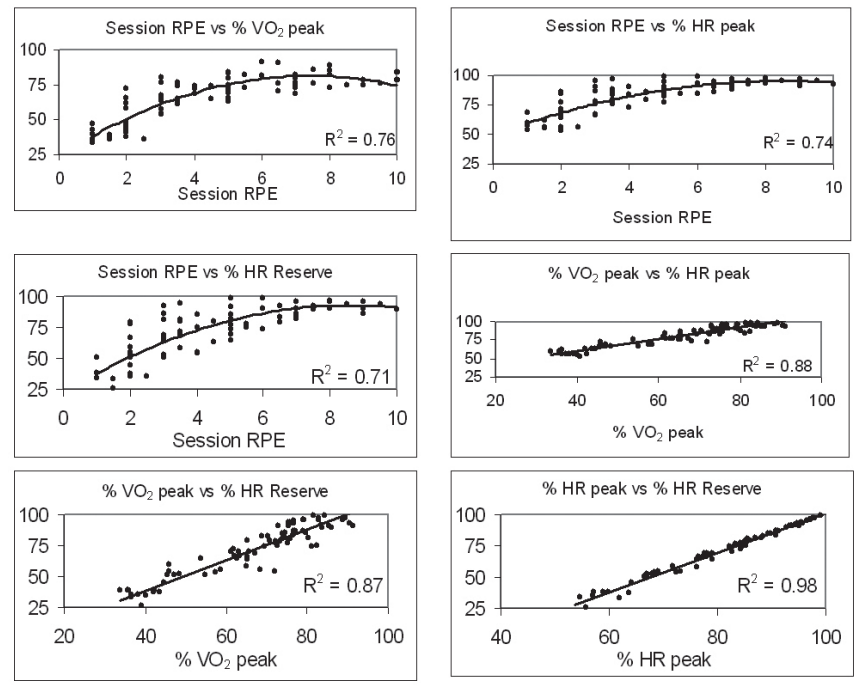

Fig. 4. Scatter plots of individual responses of Session

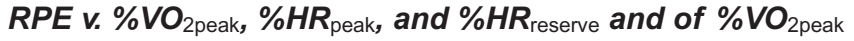
v. $\% H R_{\text {peak }}$ and $\% H R_{\text {reserve }}$ and of $\% H R_{\text {peak }} \boldsymbol{V}$. $\% H R_{\text {reserve. }}$ These plots demonstrate that although Session RPE is significantly related to objective markers of exercise intensity, it is less well related to the objective markers of exercise intensity than the objective markers are to each other.

with progressively longer performance at a fixed exercise intensity is comparable to the drift in HR during prolonged exercise. $^{8,9}$

We have not tested the Session RPE under very prolonged and exhaustive exercise such as, for example, marathon running. In a situation of competitive performance (or even severe training bouts) of a very prolonged duration, the Session RPE would approach maximal values, even though the objective exercise intensity would remain within a clearly submaximal zone. This problem remains to be explored in that it presents a fundamental problem in terms of monitoring exercise training. However, as this is also a limitation with HR-based methods of monitoring exercise intensity, this drift does not represent a limitation unique to the Session RPE method. Our underlying assumptions in studies using the Session RPE method as the intensity component of monitoring training has been to use the Session RPE to replace objective measures of exercise intensity within the training impulse (TRIMP) approach originally developed by Fitz-Clarke et al. ${ }^{5}$ and Morton et al. ${ }^{18}$ and widely used by others. $6,7,12,14,15,21$ The TRIMP approach is a very useful way of combining exercise intensity and duration into a single number representation of the stimulus for adaptation provided by any exercise bout, which has been limited by the absence of a simple method of expressing exercise intensity. During very prolonged exercise, the drift of Session RPE (or of $\mathrm{HR}$ ) acts as a multiplier of exercise duration and suggests a much larger TRIMP than might have been calculated using the RPE or HR early during the exercise bout as a marker of exercise intensity. This raises the more fundamental question implicit in the TRIMP concept related to the signal for adaptations to exercise. Clearly, exercise intensity changes the milieu internal in ways that are different from exercise duration. Until this signalling mechanism is elucidated, it may not be critical whether one or the other of the comparatively crude subjective methods of estimating exercise intensity is used.

\section{REFERENCES}

1. Balady GJ, Chaitman B, Driscoll D, et al. Recommendations for cardiovascular screening, staffing and emergency policies at health/fitness facilities: A Joint Position Statement by the American College of Sports Medicine and the American Heart Association. Med Sci Sports Exerc 1998; 30: 1009-18.

2. Borg G, Hassman P, Langerstrom M. Perceived exertion in relation to heart rate and blood lactate during arm and leg exercise. Eur J Appl Physiol 1987; 65: 679-85.

3. Borg, G, Ljunggren G, Ceci R. The increase of perceived exertion, aches and pains in the legs, heart rate and blood lactate during exercise on a bicycle ergometer. Eur J Appl Physiol 1985; 54: 343-9.

4. Day ML, McGuigan MR, Brice GA, Foster C. Monitoring work intensities during resistance training using a session RPE scale. Journal of Strength and Conditioning Research 2004; 18: 353-8.

5. Fitz-Clarke JR, Morton RH, Banister EW. Optimizing athletic performance by influence curves. J Appl Physiol 1991; 71: 1151-8.

6. Foster $\mathrm{C}$. Monitoring training in athletes with reference to overtraining syndrome. Med Sci Sports Exerc 1997; 30: 1164-8.

7. Foster C, Daines E, Hector L, Snyder A, Welsh R. Athletic performance in relation to training load. Wisconsin Medical Journal 1996; 95: 370-4

8. Foster $\mathrm{C}$, Florhaug J, Franklin J, et al. A new approach to monitoring exercise training. Journal of Strength and Conditioning Research 2001; 15: 109-15.

9. Foster C, Florhaug J, Hrovatin L. Monitoring of athletic training. Vlaams Tijdschrift voor Sportgeneeskunde en Sportwetenshappen 1999; 80: 47-54.

10. Foster C, Hector L, Welsh R, Schrager M, Green M, Snyder A. Effects of specific versus cross-training on running performance. Eur J Appl Physiol 1995; 70: 367-72.

11. Foster C, Heimann K, Esten P, Brice G, Porcari J. Differences in perceptions of training by coaches and athletes. South African Journal of Sports Medicine 2001; 8: 3-7.

12. Foster C, Hoyos J, Earnest C, Lucia A. Regulation of energy expenditure during prolonged athletic competition. Med Sci Sports Exerc 2005; 37: 670-5.

13. Impellizzeri FM, Rampinni E, Coutts AJ, Sassi A, Marcora SM. Use of RPEbased training load in soccer. Med Sci Sports Exerc 2004; 36: 1042-7.

14. Lucia A, Hoyos J, Carvajal A, Chicharro JL. Heart rate response to professional road cycling: The Tour de France. Int J Sports Med 1999; 20: 167-72.

15. Lucia A, Hoyos J, Santally A, Earnest C, Chicharro JL. Tour de France vs Vuelta a Espana: Which is harder? Med Sci Sports Exerc 2003; 35: 872-8.

16. McGuigan MR, Egan AD, Foster C. Salivary cortisol responses and perceived exertion during high intensity and low intensity bouts of resistance exercise. Journal of Sport Science and Medicine 2004; 3: 8-15.

17. McGuigan MR, Foster C. A new approach to monitoring resistance training Strength and Conditioning Journal 2004; 26: 42-7.

18. Morton RH, Fitz-Clarke JR, Banister EW. Modeling human performance in running. J Appl Physiol 1990; 69: 1171-7.

19. Noble B, Robertson R. The Borg scale: development, administration, and experimental use. In: Washburn R, Mittelmeiner K, eds. Perceived Exertion. Champaign, III: Human Kinetics Publishers, 1996: 59-92.

20. Pandolf K. Advances in the study and application of perceived exertion. Exerc Sport Sci Rev 1983; 11: 118-58.

21. Seiler KS, Kjerland GO. Quantifying training intensity distribution in elite endurance athletes: Is there evidence for an 'optimal' distribution. Scandinavian Journal of Medicine and Science in Sports (in press).

22. Skinner J, Hutsler R, Bergsteinova V, Buskirk E. The validity and reliability of a rating scale of perceived exertion. Med Sci Sports 1973; 5: 94-6.

23. Sweet TW, Foster C, McGuigan MR, Brice G. Quantitation of resistance training using the session RPE method. Journal of Strength and Conditioning Research 2004; 18: 796-802. 